\title{
Coexistence of Acute Promyelocytic Leukemia and Primary Plasma Cell Leukemia: Careful Consideration in Diagnosis
}

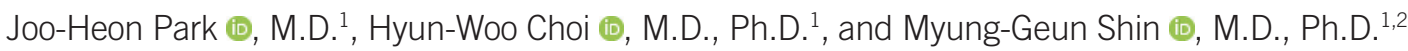

${ }^{1}$ Departments of Laboratory Medicine, Chonnam National University Medical School and Chonnam National University Hwasun Hospital, Hwasun, Korea; ${ }^{2}$ Brain Korea 21 Plus Project, Chonnam National University Medical School, Gwangju, Korea

\section{Dear Editor,}

Acute promyelocytic leukemia (APL) is frequently associated with disseminated intravascular coagulation (DIC) and hemorrhage, which contributes to its high mortality rate [1]. Molecular confirmation for identifying a $P M L-R A R A$ rearrangement has to be performed when APL is suspected. In rare cases, APL coexists with other hematologic malignancies, and in such cases, the concomitant malignant cells could be underestimated [2-4]. We report a case of APL coexisting with primary plasma cell leukemia (PCL) without any history of chemotherapy. The Institutional Review Board of Chonnam National University Hwasun Hospital (CNUHH), Hwasun, Korea (CNUHH-2021-119) approved this study and granted a waiver of informed consent because of its retrospective nature.

A 61-year-old man presented with lower back pain, exertional dyspnea, and buccal-mucosal bleeding at the hemato-oncology department of CNUHH on October 2013. He had no history of disease or treatment. A complete blood count revealed white blood cell (WBC) counts of $39.4 \times 10^{9} / \mathrm{L}$; Hb, $63 \mathrm{~g} / \mathrm{L}$; and platelet counts of $22 \times 10^{9} / \mathrm{L}$. Blood chemistry results were as follows: lactate dehydrogenase, $811 \mathrm{IU} / \mathrm{L}$ (reference interval [RI], 218472); serum creatinine, $2.0 \mathrm{mg} / \mathrm{dL}$ (RI, 0.5-1.3); total calcium, 108 mg/L (RI, 84-102); albumin, 2.7 g/dL (RI, 3.1-5.2); total protein, $6.5 \mathrm{~g} / \mathrm{dL}$ (RI, 5.8-8.1); and $\beta 2$-microglobulin, 36,675 $\mu \mathrm{g} / \mathrm{L}(\mathrm{RI}, 970-2640)$. The patient's prolonged and elevated coagulation profiles suggested DIC.

Peripheral blood (PB) and bone marrow (BM) aspirates revealed an acute leukemic state with $80 \%$ abnormal hypogranular promyelocytes having kidney-shaped or bilobed nuclei (Fig. 1A, B). A few faggot cells were also observed. In the scattergram of initial flow-cytometric analysis (Cytomics FC-500 Flow Cytometer and Kaluza Analysis Software, Beckman Coulter, Miami, FL, USA), we observed a large population of immature cells and small populations of lymphocytes and granulocytes, as seen in other leukemias. The clonal cell population stained weakly positive for myeloperoxidase (MPO) and negative for CD13, CD33, CD117, CD34, and HLA-DR. Multiplex reverse transcription PCR (HemaVision kit; DNA Technology, Aarhus, Denmark) revealed the presence of a PML-RARA rearrangement, and a conventional cytogenetic analysis demonstrated 46,XY,t(15;17)(q22;q12)[12]/ 46,XY[8] (Fig. 1C). The patient was treated with idarubicin plus all-trans retinoic acid for 40 days, and both $\mathrm{t}(15 ; 17)$ and the PMLRARA rearrangement were undetectable at follow-up.

The WBC count remained high, and the abnormal cells persisted on the PB smears. We performed follow-up BM studies 40 days after diagnosis. Flow-cytometric analysis revealed a mono-
Received: March 19, 2021

Revision received: June 15, 2021

Accepted: September 16, 2021

Corresponding author: Myung-Geun Shin, M.D, Ph.D.

Department of Laboratory Medicine, Chonnam National University Hwasun Hospital, 322 Seoyang-ro, Hwasun-eup, Hwasun-gun, Jeollanam-do 58128, Korea

Tel: +82-61-379-7950, Fax: +82-61-379-7984

E-mail:mgshin@chonnam.ac.kr 

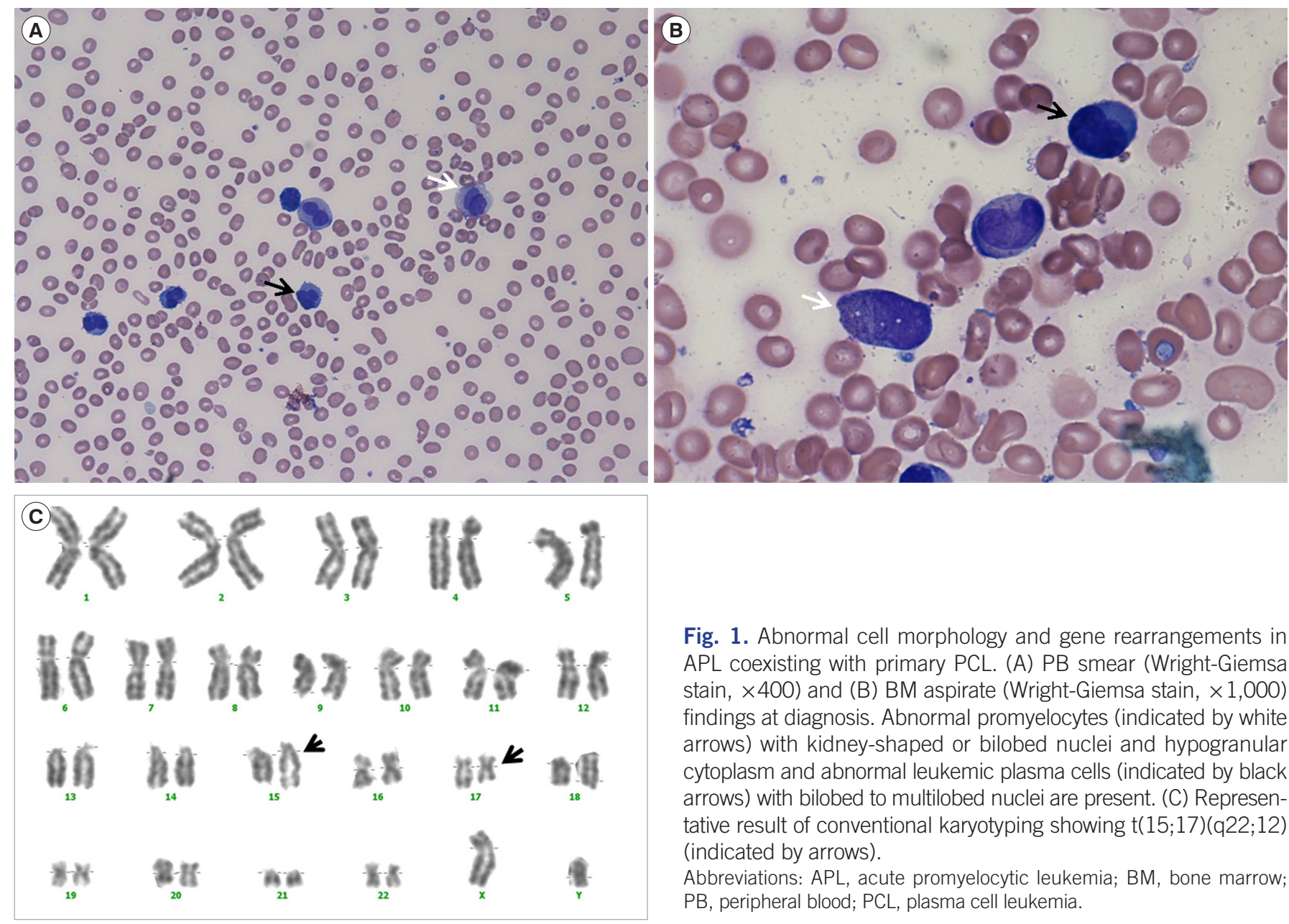

Fig. 1. Abnormal cell morphology and gene rearrangements in APL coexisting with primary PCL. (A) PB smear (Wright-Giemsa stain, $\times 400$ ) and (B) BM aspirate (Wright-Giemsa stain, $\times 1,000$ ) findings at diagnosis. Abnormal promyelocytes (indicated by white arrows) with kidney-shaped or bilobed nuclei and hypogranular cytoplasm and abnormal leukemic plasma cells (indicated by black arrows) with bilobed to multilobed nuclei are present. (C) Representative result of conventional karyotyping showing $\mathrm{t}(15 ; 17)(\mathrm{q} 22 ; 12)$ (indicated by arrows).

Abbreviations: APL, acute promyelocytic leukemia; BM, bone marrow; $\mathrm{PB}$, peripheral blood; PCL, plasma cell leukemia.

clonal population of plasma cells positive for CD138 and lambda and negative for CD56. Further investigation of the monoclonal protein revealed only lambda light chain, with a kappa/lambda ratio of 0.079 (RI, 0.26-1.65). BM sections revealed compact cellularity with plasma cells positive for CD138 and lambda light chain in immunohistochemical staining (IHCS) (Fig. 2A-C). We reviewed the results of the analyses performed for diagnosis and realized that APL and PCL coexisted. Approximately $80 \%$ of the abnormal hypogranular promyelocytes in the $\mathrm{PB}$ and $\mathrm{BM}$ were found to be abnormal plasma cells. BM sections revealed compact infiltration of two abnormal populations (CD138+ and MPO+ cells) on dual-color IHCS (Fig. 2D). We could also identify the IGH rearrangement in PCL cells by fluorescence in situ hybridization (Vysis LSI IGH Dual Color, Break Apart Rearrangement Probe; Abbott Molecular, Des Plaines, IL, USA). The patient was then treated with bortezomib plus prednisolone. During the second cycle, his general condition deteriorated such that he could no longer tolerate the chemotherapy. He was transferred to an- other hospital at his request.

The coexistence of acute leukemia and plasma cell myeloma (PCM) in a patient with no history of chemotherapy has rarely been reported [5]. The types of acute leukemia include acute myelomonocytic leukemia or acute monocytic leukemia [5]. Recently, cases of APL coexisting with PCM have been reported [2, 3]. To the best of our knowledge, this is the first reported case of coexistence of APL and primary PCL.

Primary PCL, found in $2 \%-4 \%$ of myeloma cases, is a highly aggressive disease associated with poor prognosis [6]. PCM and $\mathrm{PCL}$ are in the same disease entity; however, the differences in tumor growth and disease progression suggest that primary PCL has distinct pathogenesis [7]. The marked differences in chromosomal imbalance between PCM and PCL, which may explain the different clinical manifestations of these two disorders, has also been reported [8]. In patients of advanced age and/or with comorbidities, the recommended treatment for primary PCL is a bortezomib-based regimen, and the consolidation and mainte- 


\section{ANNALS OF
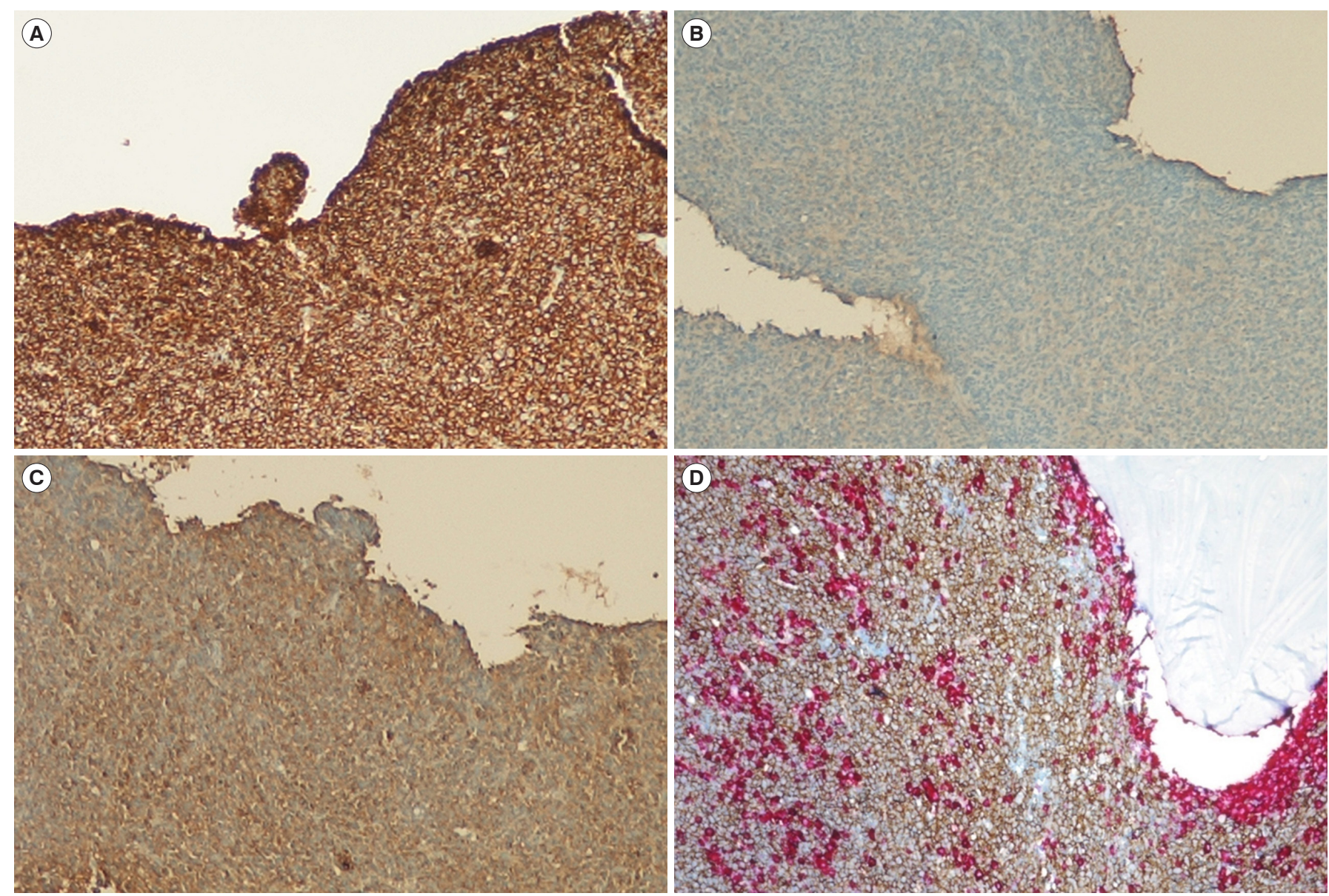

Fig. 2. IHCS of BM section at the time of follow-up evaluation and additional initial diagnosis $(\times 100)$. At follow-up, promyelocytes were depleted, and plasma leukemic cells compactly filled the entire marrow. (A) Dual-color IHCS of MPO and CD138, (B) kappa light chain, and (C) lambda light chain. (D) Dual-color IHCS of a BM section at diagnosis revealed the coexistence of MPO+ promyelocytes (red-colored, cytoplasm) and CD138+ plasma cells (brown-colored, membrane).

Abbreviations: BM, bone marrow; IHCS, immunohistochemical staining; MPO, myeloperoxidase.

nance therapy after induction therapy is crucial [9]. However, there are no established management strategies for acute leukemia cases because they are rare.

When myeloproliferative neoplasm (MPN) and PCM coexist, the management strategy is focused on treating the overt PCM. Agents, such as thalidomide or lenalidomide, which are effective for treating MPN as well as PCM, are recommended [10]. In previous studies on coexisting acute leukemia and PCM, leukemic and myeloma cells responded differently to chemotherapeutic agents, which may be because the two neoplasms develop from different cell lineages and have different pathogenetic mechanisms [3-5].

APL is clinically suspected when abnormal promyelocytes are predominant with DIC. Abnormal promyelocytes are exceedingly difficult to identify, and in addition to the typical (hypergranular) form, they can present in a microgranular (hypogranular) form, which may be confused with other cell lineages. As shown in the present case, leukemic plasma cells also have irregular (kidney-shaped, bilobed, or multilobed) nuclei and can even contain condensed or crystallized cytoplasmic Ig, resembling abnormal promyelocytes. This suggests that a comprehensive evaluation of the various possibilities with careful decision-making is necessary for the APL diagnosis, especially when cell morphology or other laboratory results are atypical. The limitation of this case study is that next-generation sequencing could not be performed because it was conducted before the establishment of next-generation sequencing at our institution. APL with DIC is a medical emergency that needs to be diagnosed and treated as soon as possible. The possibility of coexistence of other hematologic diseases or abnormalities should always be considered, even when the diagnosis of APL is confirmed by the presence of a PML-RARA rearrangement. 


\section{ACKNOWLEDGEMENTS}

None.

\section{AUTHOR CONTRIBUTIONS}

Park JH and Choi HW conceived and designed the study and collected and analyzed the data. Park JH, Choi HW, and Shin MG wrote the final manuscript. All authors have accepted their responsibility for the entire content of this manuscript and approved the submission.

\section{CONFLICTS OF INTEREST}

None declared.

\section{RESEARCH FUNDING}

None declared.

\section{ORCID}

Joo-Heon Park

Hyun-Woo Choi

https://orcid.org/0000-0002-6842-0234

Myung-Geun Shin

\section{REFERENCES}

1. Wang ZY and Chen Z. Acute promyelocytic leukemia: from highly fatal to highly curable. Blood 2008;111:2505-15.

2. Schafernak KT and Khanani FA. Simultaneous presentation of plasma cell myeloma and acute promyelocytic leukemia. Blood 2013;122:3861.

3. Lim J, Kwon GC, Koo SH, Song IC, Kim J. A case of acute promyelocytic leukemia concomitant with plasma cell myeloma. Ann Lab Med 2014 34:152-4.

4. Dunkley S, Gibson J, lland H, Li C, Joshua D. Acute promyelocytic leukaemia complicating multiple myeloma: evidence of different cell lineages. Leuk Lymphoma 1999;35:623-6.

5. Luca DC and Almanaseer IY. Simultaneous presentation of multiple myeloma and acute monocytic leukemia. Arch Pathol Lab Med 2003;127: 1506-8.

6. Swerdlow SH, Campo E, et al. eds. WHO classification of tumours of haematopoietic and lymphoid tissues. Revised 4th ed. Lyon: International Agency for Research on Cancer, 2017:250.

7. Schinke C, Boyle EM, Ashby C, Wang Y, Lyzogubov V, Wardell C, et al. Genomic analysis of primary plasma cell leukemia reveals complex structural alterations and high-risk mutational patterns. Blood Cancer J 2020; 10:70.

8. Gutiérrez NC, Hernández JM, García JL, Cañizo MC, González M, Hernández J, et al. Differences in genetic changes between multiple myeloma and plasma cell leukemia demonstrated by comparative genomic hybridization. Leukemia 2001;15:840-5.

9. van de Donk NW, Lokhorst HM, Anderson KC, Richardson PG. How I treat plasma cell leukemia. Blood 2012;120:2376-89.

10. Malhotra J, Kremyanskaya M, Schorr E, Hoffman R, Mascarenhas J. Coexistence of myeloproliferative neoplasm and plasma-cell dyscrasia. Clin Lymphoma Myeloma Leuk 2014;14:31-6. 